\title{
EFEITO DO PREPARO DO SOLO E RESÍDUO DA COLHEITA DE CANA-DE-AÇÚCAR SOBRE A EMISSÃO DE $\mathrm{CO}_{2}{ }^{(1)}$
}

\author{
Mara Regina Moitinho(2), Milton Parron Padovan ${ }^{(3)}$, Alan Rodrigo Panosso $^{(4)}$ \& Newton \\ La Scala Jr $\mathbf{r}^{(5)}$
}

\begin{abstract}
RESUMO
O solo é um dos principais compartimentos de carbono no ecossistema terrestre, capaz de armazenar quantidades expressivas desse elemento e, portanto, a compreensão dos fatores que contribuem para as perdas de $\mathrm{CO}_{2} \mathrm{em}$ solos agrícolas é fundamental para determinar estratégias de redução das emissões desse gás e ajudar a mitigar o efeito estufa. $O$ objetivo deste estudo foi investigar o efeito do preparo do solo e da deposição de resíduos da cultura da cana-de-açúcar na emissão de $\mathrm{CO}_{2}$, temperatura e umidade do solo, durante a reforma do canavial, ao longo de um período de 15 dias. Os manejos avaliados foram: sem preparo do solo e mantendo os resíduos da colheita sobre a superfície do solo (SPCR); sem preparo do solo e sem resíduo (SPSR) e com preparo do solo e sem resíduo (CPSR). A menor média de emissão de $\mathrm{CO}_{2}$ do solo $\left(\mathrm{FCO}_{2}\right)$ foi observada no manejo SPCR $\left(2,16 \mu \mathrm{mol} \mathrm{m} \mathrm{m}^{-2} \mathrm{~s}^{-1}\right)$, quando comparado aos manejos SPSR $\left(2,90 \mu \mathrm{mol} \mathrm{m}^{-2} \mathrm{~s}^{-1}\right)$ e CPSR $\left(3,22 \mu \mathrm{mol} \mathrm{m} \mathrm{m}^{-2} \mathrm{~s}^{-1}\right)$, indicando que as maiores umidades e menores variações da temperatura do solo, observadas em SPCR, foram os fatores responsáveis por tal diminuição. Durante o período de estudo, a menor média diária da $\mathrm{FCO}_{2}$ foi registrada em SPCR $\left(1,28 \mu \mathrm{mol} \mathrm{m}{ }^{-2} \mathrm{~s}^{-1}\right)$ e a maior em CPSR $\left(6,08 \mu \mathrm{mol} \mathrm{m} \mathrm{m}^{-2} \mathrm{~s}^{-1}\right)$, após a ocorrência de chuvas. A menor perda de $\mathrm{C}-\mathrm{CO}_{2}$ do solo foi observada no manejo SPCR $\left(367 \mathrm{~kg} \mathrm{ha}^{-1} \mathrm{de}\right.$ C-CO $\left.\mathrm{C}_{2}\right)$, diferindo significativamente $(\mathrm{p}<0,05)$ dos manejos: SPSR $\left(502 \mathrm{~kg} \mathrm{ha}^{-1} \mathrm{de} \mathrm{C}\right.$ $\mathrm{CO}_{2}$ ) e CPSR (535 $\mathrm{kg} \mathrm{ha}^{-1}$ de C-CO $\mathrm{CO}_{2}$. A umidade do solo foi a variável que apresentou valores mais diferenciados entre os manejos, sendo positivamente correlacionada $(\mathrm{r}=0,55 ; \mathrm{p}<0,05)$ com as variações temporais da emissão de $\mathrm{CO}_{2}$ nos manejos SPCR e CPSR. Em adição, a temperatura do solo diferiu $(p<0,05)$ somente no manejo SPCR $\left(24^{\circ} \mathrm{C}\right)$, quando comparada aos manejos SPSR $\left(26^{\circ} \mathrm{C}\right)$ e CPSR $\left(26,5^{\circ} \mathrm{C}\right)$,
\end{abstract}

\footnotetext{
(1) Parte da Dissertação de Mestrado do primeiro autor. Recebido para publicação em 7 de fevereiro de 2013 e aprovado em 20 de agosto de 2013.

(2) Mestranda em Agronomia, Programa de Pós-Graduação em Ciência do Solo, UNESP/Jaboticabal. Via de Acesso Prof. Paulo Donato Castellane, s/n. CEP 14884-900 Jaboticabal (SP). E-mail: maramoitinho@gmail.com

(3) Pesquisador da Embrapa Agropecuária Oeste. BR 163, km 253,6. Caixa Postal 449. CEP 79804-970 Dourados (MS). E-mail: milton.padovan@embrapa.br

(4) Professor Assistente Doutor do Departamento de Matemática, UNESP/Ilha Solteira, Avenida Brasil, 56. CEP 15385-000 Ilha Solteira (SP). E-mail: alanrp@mat.feis.unesp.br

(5) Professor Adjunto do Departamento de Ciências Exatas, UNESP/Jaboticabal. E-mail: lascala@fcav.unesp.br
} 
sugerindo que, para as condições deste estudo, o resíduo da cana-de-açúcar retido sobre a superfície propiciou uma temperatura do solo, em média, $2{ }^{\circ} \mathrm{C}$ mais amena.

Termos de indexação: respiração do solo, manejo da cana-de-açúcar, temperatura e umidade do solo.

\title{
SUMMARY: EFFECT OF SOIL TILLAGE AND SUGARCANE TRASH ON $\mathrm{CO}_{2}$ EMISSION
}

\begin{abstract}
The soil is one of the main C pools in terrestrial ecosystem, capable of storing significant $\mathrm{C}$ amounts. Therefore, understanding the factors that contribute to the loss of $\mathrm{CO}_{2}$ from agricultural soils is critical to determine strategies reducing emissions of this gas and help mitigate the greenhouse effect. The purpose of this study was to investigate the effect of soil tillage and sugarcane trash on $\mathrm{CO}_{2}$ emissions, temperature and soil moisture during sugarcane (re)planting, over a study period of 15 days. The following managements were evaluated: notillage with crop residues left on the soil surface (NTR); without tillage and without residue (NTNR) and tillage with no residue (TNR). The average soil $\mathrm{CO}_{2}$ emission $\left(\mathrm{FCO}_{2}\right)$ was lowest in NTR $\left(2.16 \mu \mathrm{mol} \mathrm{m}{ }^{-2} \mathrm{~s}^{-1}\right)$, compared to the managements NTNR $\left(2.90 \mu \mathrm{mol} \mathrm{m} \mathrm{m}^{-2} \mathrm{~s}^{-1}\right)$ and TNR $\left(3.22 \mu \mathrm{mol} \mathrm{m} \mathrm{m}^{-2} \mathrm{~s}^{-1}\right)$, indicating that the higher moisture and lower soil temperature variations observed in NTR were responsible for this decrease. During the study period, the lowest daily average $\mathrm{FCO}_{2}$ was recorded in NTR $\left(1.28 \mu \mathrm{mol} \mathrm{m}^{-2} \mathrm{~s}^{-1}\right)$, and the highest in TNR $\left(6.08 \mathrm{umol} \mathrm{m}^{-2} \mathrm{~s}^{-1}\right)$, after rainfall. A loss of soil $\mathrm{CO}_{2}$ was lowest from the management NTR $\left(367 \mathrm{~kg} \mathrm{ha}^{-1}\right.$ of $\left.\mathrm{CO}_{2-}-\mathrm{C}\right)$ and differing significantly $(p<0.05)$ from the managements NTNR $\left(502 \mathrm{~kg} \mathrm{ha}^{-1}\right.$ of $\left.\mathrm{CO}_{2^{-}} \mathrm{C}\right)$ and $\mathrm{TNR}\left(535 \mathrm{~kg} \mathrm{ha} a^{-1}\right.$ of $\left.\mathrm{CO}_{2}-\mathrm{C}\right)$. Soil moisture was the variable that differed most managements and was positively correlated $(r=0.55, p<0.05)$ with the temporal variations of $\mathrm{CO}_{2}$ emission from NTR and TNR. In addition, the soil temperature differed $(p<0.05)$ only in management NTR $\left(24^{\circ} \mathrm{C}\right)$ compared to NTNR $\left(26^{\circ} \mathrm{C}\right)$ and $\operatorname{TNR}\left(26.5^{\circ} \mathrm{C}\right)$, suggesting that under the conditions of this study, sugarcane trash left on the surface induced an average rise in the of soil temperature of $2{ }^{\circ} \mathrm{C}$.
\end{abstract}

Index terms: soil respiration, sugarcane management, soil temperature and moisture.

\section{INTRODUÇÃO}

A ocorrência de fenômenos naturais extremos como as intensas ondas de calor, as tempestades, as secas e os furacões, cada vez mais severos, tem sido apontada como consequência das mudanças ocorridas no clima global, decorrentes principalmente da emissão adicional de gases do efeito estufa, como: o dióxido de carbono $\left(\mathrm{CO}_{2}\right)$, o metano $\left(\mathrm{CH}_{4}\right)$ e o óxido nitroso $\left(\mathrm{N}_{2} \mathrm{O}\right)$. Estudos sugerindo estratégias para redução desses gases na atmosfera e mitigação do aquecimento global estão cada vez mais em foco (Cerri et al., 2009; Canellas et al., 2010; Figueiredo, 2012). Embora o potencial de aquecimento global do $\mathrm{CH}_{4}$ e do $\mathrm{N}_{2} \mathrm{O}$ ser em torno de 23 e 298 vezes maior que o de $\mathrm{CO}_{2}$, respectivamente (Cerri et al., 2007), o $\mathrm{CO}_{2}$ é o que mais contribuiu em termos relativos para o efeito estufa adicional nos últimos 200 anos, em razão da grande quantidade emitida na atmosfera (IPCC, 2007).

Algumas práticas agrícolas, como mudanças do uso do solo, a exemplo de conversão de vegetação natural para pastagem e agricultura, além de manejos do solo com intenso revolvimento, estão intimamente relacionadas a tais emissões (Roscoe et al., 2006; Dendooven et al., 2012).
No Brasil, aproximadamente $75 \%$ das emissões de $\mathrm{CO}_{2}$ são provenientes das atividades agrícolas (Cerri et al., 2009). De acordo com dados da Companhia Nacional do Abastecimento (CONAB, 2012), o país vem destacando-se mundialmente por ser o maior produtor de cana-de-açúcar (Saccharum spp.), com produção estimada em 597 milhões de toneladas de cana moída na safra de 2012/2013. A área cultivada com cana-deaçúcar que será colhida e destinada à atividade sucroalcooleira na safra de 2012/2013 está estimada em 8,5 milhões de hectares. São Paulo é o maior Estado produtor, com $52 \%$ da área plantada, sendo o Estado do Mato Grosso do Sul o quinto do ranking nacional, com 554 mil hectares cultivados com canade-açúcar (6,5\%).

Diante do crescimento acelerado desse setor e da grande preocupação com os impactos oriundos de práticas de cultivo e manejo dos solos para uso agrícola, diversos estudos têm-se concentrado nos sistemas de produção da cana-de-açúcar (La Scala et al., 2006; Panosso et al., 2009a; Silva-Olaya et al., 2013).

A cultura da cana-de-açúcar tem a capacidade de retirar da atmosfera e incorporar em sua fitomassa uma grande quantidade de $\mathrm{CO}_{2}$, em torno de $100 \mathrm{t} \mathrm{ha}^{-1} \mathrm{ano}^{-1}$, o equivalente a $30 \mathrm{t} \mathrm{ha}^{-1} \mathrm{ano}^{-1} \mathrm{de}$ 
C. Se parte desse $\mathrm{C}$ ficar acumulada no solo por meio da matéria orgânica por um longo período, o sistema passa a funcionar como coletor líquido para o sequestro de $\mathrm{CO}_{2}$ atmosférico, auxiliando na mitigação do efeito estufa (Ronquim, 2007).

Sistemas de plantio em que os resíduos culturais são retidos sobre o solo aumentam significativamente o teor de C nas camadas mais superficiais desse, quando comparados aos sistemas com preparo convencional (Canellas et al., 2010). Em média, cinco vezes mais C é acumulado no solo, em razão da adoção do sistema conservacionista (Dendooven et al., 2012). Em adição, a reforma do canavial reduz o potencial de sequestro de C nesse manejo (La Scala et al., 2012), uma vez que a perturbação física do solo durante esse período acarretará o aumento da oxidação da matéria orgânica e as perdas por erosão (Galdos et al., 2009).

As intensas atividades de preparo do solo aumentam, inicialmente, as taxas de emissão de $\mathrm{CO}_{2}$ dele para a atmosfera, pois com o rompimento dos agregados do solo, parte do $\mathrm{C}$ anteriormente protegido em seu interior é exposta à ação microbiana, tornando-o mais suscetível à mineralização (Reicosky \& Archer, 2007; Schwartz et al., 2010). Após as operações de preparo, aumenta-se a aeração do solo e, consequentemente, ocorre maior acessibilidade ao oxigênio, necessário para a microbiota (La Scala et al., 2006; Gesch et al., 2007), principal responsável pela decomposição da sua matéria orgânica (Graham et al., 2006; Lou et al., 2011). Variações estacionais com elevações nas temperaturas do solo e do ar e na umidade do solo também oferecem condições propícias para maximizar a atividade dos microrganismos desse, intensificando a taxa de degradação da matéria orgânica (Silva-Olaya et al., 2013).

Além desse efeito, no processo de emissão de $\mathrm{CO}_{2}$, particularmente, o transporte do gás do interior do solo até a superfície é governado pela equação de difusão, que por sua vez é influenciada, em primeira ordem, por alterações na temperatura e umidade do solo (Kang et al., 2003). Considerando que tanto a umidade quanto a temperatura apresentam grande variação espaçotemporal e relação direta com as condições climáticas locais (Panosso et al., 2009a), a caracterização do fluxo de $\mathrm{CO}_{2}$ do solo é um dos grandes desafios da comunidade científica em razão das incertezas relacionadas a essas medidas. Dentro desse contexto, o objetivo deste estudo foi investigar o efeito do preparo do solo e da deposição de resíduos da cultura da cana-de-açúcar na emissão de $\mathrm{CO}_{2}$, assim como seus efeitos na temperatura e umidade dele, durante a reforma do canavial, ao longo de um período de 15 dias de estudo.

\section{MATERIAL E MÉTODOS}

O estudo foi conduzido na área experimental da Embrapa Agropecuária Oeste, em Dourados, MS, localizada a $22^{\circ} 14^{\prime}$ latitude sul, $54^{\circ} 49^{\prime}$ longitude oeste de Greenwich e $452 \mathrm{~m}$ de altitude, num Latossolo Vermelho distroférrico típico textura muito argilosa (152; 104; e $744 \mathrm{~g} \mathrm{~kg}^{-1}$ de areia, silte e argila, respectivamente), conforme Embrapa (2006). A área apresenta relevo plano com declives inferiores a $3 \%$. Segundo a classificação de Thornthwaite, o clima local pode ser definido como $\mathrm{B}_{1} \mathrm{rB}_{4}{ }_{4} \mathrm{a}$ ', tipo mesotérmico úmido, com pequena ou nenhuma deficiência hídrica, sendo a evapotranspiração de verão menor que $48 \%$ da anual. A temperatura média anual varia de 20 a $22{ }^{\circ} \mathrm{C}$, com as médias dos meses mais frios e mais quentes oscilando, respectivamente, de 15 a $19{ }^{\circ} \mathrm{C}$ e de 23 a $26^{\circ} \mathrm{C}$. A precipitação pluvial média anual é de $1.550 \mathrm{~mm}$, sendo novembro, dezembro e janeiro o trimestre mais chuvoso (Amaral et al., 2000).

O plantio da cana-de-açúcar ocorreu no dia 28 de novembro de 2009. A área experimental foi composta pelas variedades RB 93-5744, RB 72-454, RB 93-5608, RB 85-5113 e SP 83-2847, espaçada de 1,2 m entrelinhas, totalizando $3.000 \mathrm{~m}^{2}$ de área plantada. A adubação foi realizada aplicando $2 \mathrm{tha}^{-1}$ de composto orgânico no sulco de plantio, imediatamente abaixo dos colmos da cana. Anteriormente ao plantio da cana, a área foi cultivada com aveia-preta (Avena stringosa) por dois anos, como adubo verde.

A operação de colheita da cana foi realizada manualmente e sem queima do canavial, entre os dias 21 e 25 de novembro de 2011. Após a colheita da cana, a área foi dividida em três parcelas experimentais de 25 x 40 m, constituindo os seguintes manejos: sem preparo do solo e com resíduos da colheita retidos sobre a superfície do solo (SPCR); sem preparo do solo e sem resíduos (SPSR); e com preparo do solo e sem resíduo (CPSR).

O preparo do solo ocorreu no dia 27 de novembro de 2011, composto por uma subsolagem, com um subsolador de sete hastes e profundidade de descompactação de $40 \mathrm{~cm}$. Na sequência, foram realizadas duas gradagens com grade aradora de 16 discos de 32", na profundidade de $20 \mathrm{~cm}$, para o revolvimento do solo.

A palha remanescente da colheita da cana-deaçúcar correspondeu a $13,5 \mathrm{t} \mathrm{ha}^{-1}$ de massa de matéria seca, com uma altura próxima a $15 \mathrm{~cm}$ de material seco deixado sobre o solo. A quantidade de palhada deixada na superfície foi caracterizada pela coleta de amostras de palha retidas dentro de $1 \mathrm{~m}^{2}$, sendo posteriormente pesadas e levadas à estufa de ventilação forçada a $65{ }^{\circ} \mathrm{C}$, até peso constante, para determinação da massa de matéria seca.

A emissão de $\mathrm{CO}_{2}$ do solo foi registrada utilizandose de um sistema portátil da companhia LI-COR (LI8100), Nebraska EUA. Em seu modo de medição, o sistema monitora as mudanças na concentração de $\mathrm{CO}_{2}$ dentro da câmara, por meio de espectroscopia de absorção óptica na região do infravermelho (IRGA Infrared Gas Analyzer). Esse é acoplado sobre tubos de PCV com diâmetro de $10 \mathrm{~cm}$ e inseridos $3 \mathrm{~cm}$ dentro do solo. Concomitantemente às leituras de emissão de 
$\mathrm{CO}_{2}$, foram conduzidas avaliações da temperatura do solo na camada de $0-20 \mathrm{~cm}$ de profundidade, utilizando-se de um termômetro (termistor portátil), que é parte integrante do sistema em que a câmara para solos é acoplada. A umidade do solo foi também medida em todos os pontos, utilizando-se de um sistema portátil TDR-Campbel® (Hydrosense TM, Campbell Scientific, Australia), que avaliou a umidade disponível do solo (\% em volume) na camada de $0-20 \mathrm{~cm}$.

Em cada parcela experimental, foram inseridos 10 tubos de PVC para as avaliações da emissão de $\mathrm{CO}_{2}$ do solo $\left(\mathrm{FCO}_{2}\right)$, sendo a temperatura do solo (Ts) e a umidade do solo (Us) também investigadas adjacentes aos tais tubos. Foram 15 dias de avaliações compreendidos entre os dias 28 de novembro e 14 de dezembro de 2011, no horário das 8 às $10 \mathrm{~h}$. Informações referentes às precipitações pluviais ocorridas na área, durante o período experimental, foram extraídas de um conjunto de dados pertencentes ao acervo da Estação Agrometeorológica da Embrapa Agropecuária Oeste, localizada próxima ao local onde o experimento foi conduzido.

Os dados foram analisados, inicialmente, por meio da estatística descritiva (média, desvio-padrão, erropadrão da média, máximo e mínimo e coeficiente de variação). A análise de variância foi realizada em delineamento inteiramente casualizado com parcelas subdivididas. Para a verificação das pressuposições do modelo, realizou-se o teste de esfericidade da matriz de variâncias e covariâncias entre tempos. A hipótese de esfericidade das matrizes de variância e covariância não foi rejeitada para todas as variáveis estudadas. As análises de variância e de correlação linear entre as variáveis foram efetuadas, utilizando o sistema SAS (SAS versão 9.1, SAS instituto, Cary, NC, EUA). As emissões acumuladas de $\mathrm{CO}_{2}$ do solo, durante todo o período de estudo, foram estimadas pelo método da área abaixo das curvas de emissão, utilizando o software R (R Development Core Team, 2011).

\section{RESULTADOS E DISCUSSÃO}

A média \pm erro-padrão da emissão de $\mathrm{CO}_{2}$ do solo $\left(\mathrm{FCO}_{2}\right)$ no manejo SPCR $\left(2,16 \pm 0,06 \mu \mathrm{mol} \mathrm{m}^{-2} \mathrm{~s}^{-1}\right)$ indicou que essa foi $34 \%$ inferior àquela registrada no manejo SPSR $\left(2,90 \pm 0,15 \mu \mathrm{mol} \mathrm{m}^{-2} \mathrm{~s}^{-1}\right)$; assim, pode-se inferir que a retirada da palha residual da cana-de-açúcar favoreceu maiores emissões de $\mathrm{CO}_{2} \mathrm{em}$ relação àquele com a presença da palha, no período estudado (Quadro 1). Panosso et al. (2011), caracterizando a $\mathrm{FCO}_{2}$ em áreas de cana crua e queimada, observaram emissões de $2,07 \pm 0,06 \mu \mathrm{mol} \mathrm{m}^{-2} \mathrm{~s}^{-1} \mathrm{no}$ sistema de cana crua equivalente a uma emissão $32 \%$ inferior àquela do sistema de cana queimada, valores semelhantes aos observados neste estudo. A manutenção dos resíduos da cultura da cana-de-açúcar sobre a superfície do solo favorece certas propriedades dele, como a redução da temperatura e a manutenção do teor de água (Ussiri \& Lal, 2009), a baixa densidade (Tominaga et al., 2002), as taxas de infiltração e a estabilidade de agregados (Graham \& Haynes, 2006), além de proporcionar significativo aumento no teor da matéria orgânica (Canellas et al., 2010).

Outros autores, estudando a emissão de $\mathrm{CO}_{2}$ do solo em áreas de cana-de-açúcar com colheita de cana crua, também observaram que a $\mathrm{FCO}_{2}$ é significativamente menor no sistema de manejo onde os resíduos são deixados sobre o solo (La Scala et al., 2006; Panosso et al., 2009a; Silva-Olaya et al., 2013; Figueiredo, 2012), sendo esse, também, o manejo que contribuiu para que maiores quantidades de $\mathrm{C}$ sejam armazenadas nas suas camadas mais superficiais (Canellas et al., 2010; La Scala et al., 2012; Thorburn et al., 2012). Resende et al. (2006) estimaram que a manutenção em torno de $15 \mathrm{t} \mathrm{ha}^{-1} \mathrm{ano}^{-1}$ de matéria seca dos resíduos da cana-de-açúcar poderia resultar em acúmulo de $\mathrm{C}$ no solo no longo prazo. Entretanto, deve-se evitar a incorporação desses resíduos ao solo, deixando-os somente sobre sua superfície, uma vez que com a incorporação há maior aeração, aumento na temperatura do solo e diminuição da umidade, decorrentes do processo de revolvimento, facilitando o acesso microbiano ao material orgânico (Tominaga et al., 2002; Roscoe et al., 2006; Silva-Olaya et al., 2013).

No manejo CPSR, o valor médio da $\mathrm{FCO}_{2}$ foi de $3,22 \pm 0,17 \mu \mathrm{mol} \mathrm{m}^{-2} \mathrm{~s}^{-1}$, ou seja, $49 \%$ superior àquele observado no manejo $\operatorname{SPCR}\left(2,16 \pm 0,06 \mu \mathrm{mol} \mathrm{m}^{-2} \mathrm{~s}^{-1}\right)$. Entretanto, o efeito do preparo do solo sobre a $\mathrm{FCO}_{2}$ pode ser determinado quando as emissões provenientes dos manejos CPSR e SPSR são comparadas. Não houve diferenças significativas entre as médias de $\mathrm{FCO}_{2}$ para esses manejos, indicando que o efeito do preparo do solo, como fator isolado, não influenciou o aumento nas taxas de emissão de $\mathrm{CO}_{2}$ desse (Quadro 1), durante o período estudado, sendo a retirada dos resíduos o fator determinante para os maiores valores médios registrados nesses manejos.

$\mathrm{Na}$ análise de variância para a emissão de $\mathrm{CO}_{2}$ do solo, foi indicado efeito não significativo $(\mathrm{p}=0,2940) \mathrm{da}$ interação entre os tratamentos e o tempo (dias de avaliação). Portanto, a $\mathrm{FCO}_{2}$ apresentou padrão similar de variabilidade temporal quando os três sistemas de manejo foram comparados. Na figura 1, são apresentadas as médias diárias da $\mathrm{FCO}_{2}$, temperatura e umidade do solo durante o período avaliado. Embora variações na $\mathrm{FCO}_{2}$ tenham sido observadas nos três manejos, após as precipitações na área de estudo, quando comparadas as médias da emissão de $\mathrm{CO}_{2}$ do solo, observa-se que o manejo CPSR foi mais sensível às variações no teor de água no solo, apresentando os maiores valores médios diários após eventos das chuvas.

O primeiro evento foi registrado no $5^{\circ}$ dia de avaliação $\left(6,08 \mu \mathrm{mol} \mathrm{m}^{-2} \mathrm{~s}^{-1}\right)$, representando aumento de $70 \%$, quando comparado à média da emissão do dia anterior $\left(3,42 \mu \mathrm{mol} \mathrm{m}{ }^{-2} \mathrm{~s}^{-1}\right)$; no $3^{0}$ dia, ocorreu 


\begin{tabular}{|c|c|c|c|c|c|c|}
\hline Tratamento & Média & DP & EP & Mínimo & Máximo & CV \% \\
\hline \multicolumn{7}{|c|}{ Emissão de $\mathrm{CO}_{2}$ do solo $\left(\mu \mathrm{mol} \mathrm{m} \mathrm{m}^{-2} \mathrm{~s}^{-1}\right)$} \\
\hline SPCR & 2,16 & 0,66 & 0,06 & 0,54 & 4,26 & 30,55 \\
\hline SPSR & 2,90 & 1,59 & 0,15 & 0,19 & 11,89 & 54,83 \\
\hline CPSR & 3,22 & 1,87 & 0,17 & 0,81 & 13,10 & 58,07 \\
\hline \multicolumn{7}{|c|}{ Temperatura do solo $\left({ }^{\circ} \mathrm{C}\right)$} \\
\hline SPCR & 24,28 & 0,76 & 0,07 & 22,90 & 29,00 & 3,13 \\
\hline SPSR & 26,26 & 1,05 & 0,10 & 23,70 & 29,64 & 4,00 \\
\hline CPSR & 26,54 & 1,53 & 0,14 & 23,34 & 31,54 & 5,76 \\
\hline \multicolumn{7}{|c|}{ Umidade do solo (\% de volume) } \\
\hline SPCR & 44,13 & 9,04 & 0,83 & 27,00 & 67,00 & 20,48 \\
\hline SPSR & 36,13 & 12,31 & 1,13 & 19,00 & 58,00 & 34,07 \\
\hline CPSR & 27,50 & 8,58 & 0,79 & 14,00 & 66,00 & 31,20 \\
\hline
\end{tabular}

$\mathrm{n}$ = 120; DP: desvio-padrão; EP: erro-padrão da média; e CV: coeficiente de variação.

uma chuva de 10,2 mm na região, após um período de 12 dias sem chuvas. O segundo maior valor foi observado no $11^{\circ}$ dia $\left(4,34 \mu \mathrm{mol} \mathrm{m}{ }^{-2} \mathrm{~s}^{-1}\right)$, ocorrendo após a uma precipitação acumulada no $11^{\circ} ; 8^{\circ}$ e $9^{\circ}$ dias de avaliações, totalizando 33,2 mm (Figura 1a).

Varella et al. (2004) observaram resultados semelhantes em estudos desenvolvidos em solos com pastagem de braquiária em áreas de cerrado na região central do Brasil, onde o fluxo de $\mathrm{CO}_{2}$ oscilou de 1,6 para $6,2 \mu \mathrm{mol} \mathrm{m}{ }^{-2} \mathrm{~s}^{-1}$, depois da adição artificial de água no solo por simulação de chuvas. Panosso et al. (2009a), em estudos conduzidos em área de cana queimada, no interior do Estado de São Paulo, também observaram aumento significativo na $\mathrm{FCO}_{2}$, após uma precipitação de $21 \mathrm{~mm}$. Morell et al. (2010), estudando o efeito no curto prazo de chuvas sobre o fluxo de $\mathrm{CO}_{2}$, em diferentes sistemas de manejo do solo, notaram que a $\mathrm{FCO}_{2}$ foi significativamente maior nos sistemas de cultivo mínimo e plantio direto, quando comparados sistemas de preparo convencional do solo, dentro de um período de três dias depois da chuva.

A desestruturação física do solo, associada ao preparo, pode também ter influenciado as maiores médias diárias da $\mathrm{FCO}_{2}$, principalmente nos primeiros dias após o preparo ( $1^{\circ}$ ao $6^{\circ}$ dia), (Figura 1a). Aumentos na $\mathrm{FCO}_{2}$ induzido pelo preparo têm sido associados à intensidade desse, sendo fator determinante da variabilidade temporal do fluxo de $\mathrm{CO}_{2}$ do solo em períodos curtos (La Scala et al., 2009; Morell et al., 2010). Além desses efeitos, o preparo deixa o solo suscetível ao processo de erosão, o que resulta, assim, em maior perda de C (Martínez-Mena et al., 2012).

A retirada dos resíduos de palha da superfície do solo acelera esse processo (Gaiser et al., 2008). Em adição, a manutenção dos resíduos eleva a rugosidade do solo, sendo associada à baixa movimentação, favorecendo um controle mais eficaz da erosão hídrica, pois dissipa a energia da chuva e, em parte, da enxurrada, protegendo o solo do selamento superficial (Martins Filho et al., 2009).

A temperatura do solo (Ts) no manejo SPCR apresentou o valor médio de $24,2{ }^{\circ} \mathrm{C}$, valor significativamente inferior $(\mathrm{p}<0,05)$ àqueles observados para SPSR e CPSR, com 26,2 e $26,5{ }^{\circ} \mathrm{C}$, respectivamente (Quadro 1). O efeito do resíduo da cana-de-açúcar sobre os menores valores médios e diários referentes à Ts são observados quando comparados os manejos SPCR e SPSR, em que mais uma vez o manejo SPCR se destacou, apresentando menores variações durante o período de estudo, variando entre 23 e $25{ }^{\circ} \mathrm{C}$, uma vez que, no manejo SPSR, esses valores estão entre 24 e $28^{\circ} \mathrm{C}$ (Figura 1).

Dependendo da quantidade e da distribuição da cobertura vegetal no solo, a temperatura da superfície pode ser reduzida em até $4{ }^{\circ} \mathrm{C}$; com uma cobertura de aproximadamente $70 \%$, a taxa de evaporação do solo reduz para cerca de $25 \%$ (Lou et al., 2011). O efeito proporcionado pela cobertura do solo na redução da Ts é fator controlador da emissão de $\mathrm{CO}_{2}$, uma vez que a atividade microbiana é acelerada com aumentos na temperatura do solo e, consequentemente, a taxa de mineralização do C é maior (Ussiri \& Lal, 2009).

No manejo CPSR, a Ts variou entre 24 e $29,5^{\circ} \mathrm{C}$, embora esse manejo tenha registrado inicialmente as maiores médias entre os manejos avaliados; nos cinco últimos dias de medições, seus valores médios diários ficaram próximos aos registrados no manejo SPSR. A elevação inicial da Ts, possivelmente, está relacionada ao preparo do solo.

La Scala et al. (2006), investigando a emissão de $\mathrm{CO}_{2}$ do solo em áreas de cana-de-açúcar sobre 

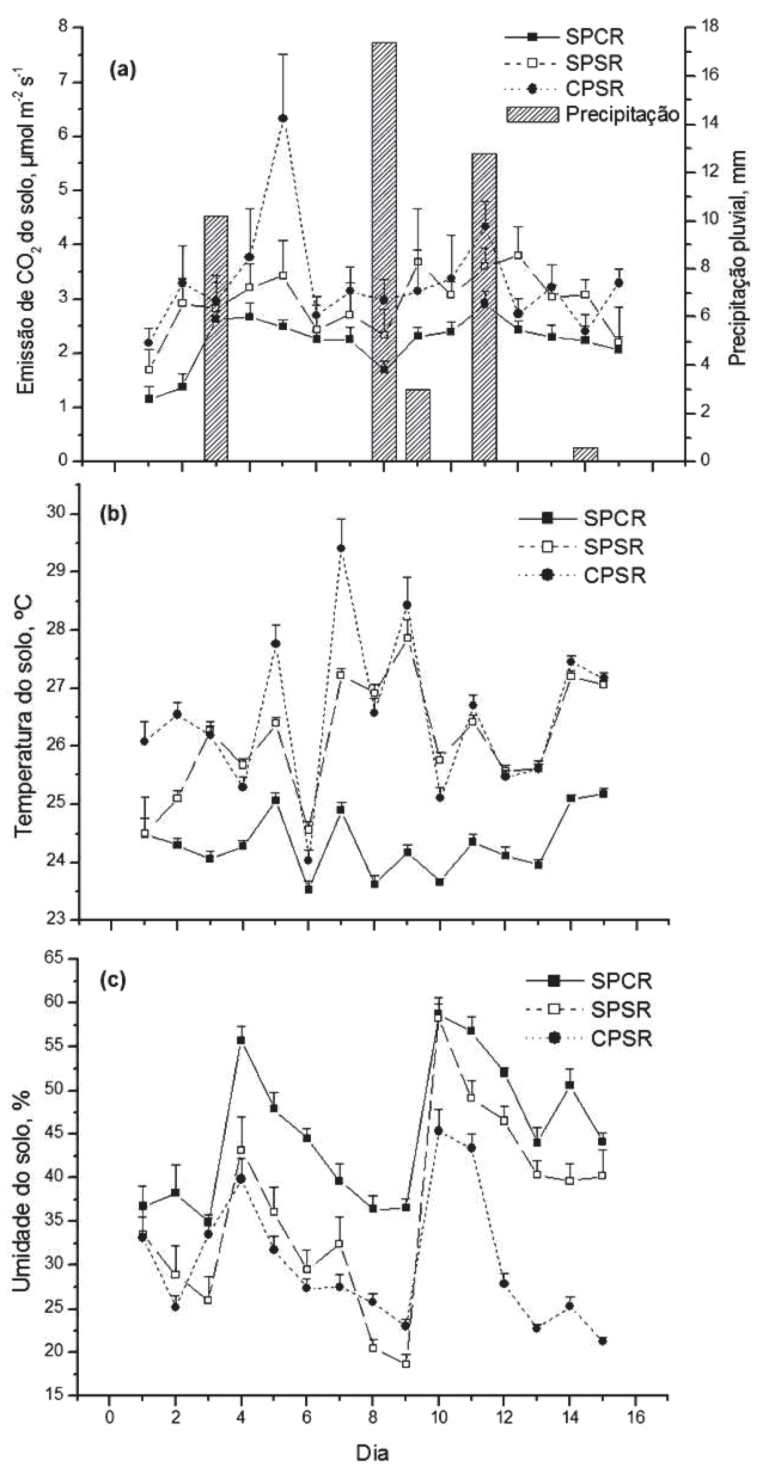

Figura 1. Emissão de $\mathrm{CO}_{2}$ do solo e precipitações pluviais ocorridas no período de estudo (a), temperatura do solo (b) e umidade do solo (c) com metade das barras de erro-padrão nos diferentes manejos: sem preparo do solo e com resíduo (SPCR), sem preparo e sem resíduo (SPSR) e com preparo e sem resíduo (SPCR).

diferentes sistemas de manejo no interior do Estado de São Paulo, também observaram valores de temperatura média do solo $2,5^{\circ} \mathrm{C}$ mais elevada no sistema onde o solo sofreu preparo.

Silva-Olaya et al. (2013), em estudo semelhante desenvolvido no nordeste do Estado de São Paulo, avaliando diferentes manejos e preparos do solo durante a reforma do canavial, observaram nos 10 dias iniciais de avaliações valores levemente superiores da temperatura do solo $\left(20\right.$ a $\left.23{ }^{\circ} \mathrm{C}\right)$, coincidindo com a realização do preparo do solo e eventos chuvosos na área.

Para a temperatura e umidade do solo, a análise de variância indicou significância $(p<0,0001)$ da interação entre os manejos e o tempo; portanto, o padrão de variabilidade temporal não foi o mesmo para os manejos. A umidade média do solo (Us) foi a propriedade que apresentou maior variabilidade temporal nos três manejos ( $\mathrm{p}<0,0001)$; a maior média de umidade do solo foi observada no manejo SPCR (44,13\%), seguido pelo SPSR (36,13\%) e CPSR (27,50 \%) (Quadro 1).

A manutenção de resíduos de colheita na superfície do solo preserva a umidade do solo, proporcionando relativo isolamento térmico (Ussiri \& Lal, 2009), reduzindo as temperaturas máximas diárias e elevando as temperaturas mínimas, em relação a solos sem cobertura vegetal (Tominaga et al., 2002). Pelas mesmas razões, nota-se que o menor valor médio da Us registrado no CPSR está possivelmente relacionado com o preparo do solo e sua subsequente aeração, pois alterações em sua estrutura física acarretam mudanças na evapotranspiração (Schwartz et al., 2010), influenciando o regime de temperatura e acelerando o processo de secagem do solo (Silva-Olaya et al., 2013).

A análise de correlação linear entre a $\mathrm{FCO}_{2}$ e a Us foi utilizada para avaliar a possível relação entre elas (Figura 2). Foi observado índice de correlação positivo e significativo $(r=0,55 ; p<0,05)$ entre essas variáveis, nos manejos SPCR e CPSR, e valor não significativo $(p<0,10)$, para o manejo SPSR. Esses resultados corroboram com aqueles obtidos por La Scala et al. (2006), em estudo semelhante, em que a relação entre a $\mathrm{FCO}_{2}$ e Us foi descrita por função linear com $\mathrm{r}=0,57$ no sistema de plantio direto e $\mathrm{r}=0,59$, no sistema de preparo convencional do solo. Os autores ressaltaram que em razão da grande variação apresentada pela umidade do solo essa se torna fator determinante da intensidade da atividade microbiológica e, portanto, contribui efetivamente para o processo de emissão de $\mathrm{CO}_{2}$ dele para a atmosfera. Panosso et al. (2009b) também observaram correlação linear positiva e significativa entre a emissão e umidade do solo logo após irrigação artificial, nos solos desprovidos de vegetação.

Os resultados deste estudo indicaram que o teor de água no solo, durante o experimento, variou dentro de um intervalo em que seu aumento resultou em elevação na atividade microbiana, sem, contudo, limitar a oxigenação do solo.

As análises de regressão linear entre a $\mathrm{FCO}_{2} \mathrm{eTs}$, para cada um dos manejos avaliados, não foram significativas $(p<0,10)$, pois a baixa variabilidade da Ts verificada pelo coeficiente de variação (Quadro 1), possivelmente, contribuiu para a ausência de correlação entre as duas variáveis.

Os valores obtidos da emissão total de $\mathrm{C}-\mathrm{CO}_{2}$ durante os 15 dias de estudo, nos diferentes manejos avaliados, demonstraram que o sistema de manejo sem preparo e com resíduos culturais da cana (SPCR) foi responsável pela menor perda de $\mathrm{C}-\mathrm{CO}_{2}$ do solo $\left(367,23 \pm 10,57 \mathrm{~kg} \mathrm{ha}^{-1}\right.$ de $\left.\mathrm{C}-\mathrm{CO}_{2}\right)$, diferindo dos manejos: SPSR $\left(501,75 \pm 29,42 \mathrm{~kg} \mathrm{ha}^{-1}\right.$ de C-CO $\left.\mathrm{CO}_{2}\right) \mathrm{e}$ CPSR (534,84 $\pm 33,81 \mathrm{~kg} \mathrm{ha}^{-1}$ de C-CO ${ }_{2}$ ) (Figura 3). 


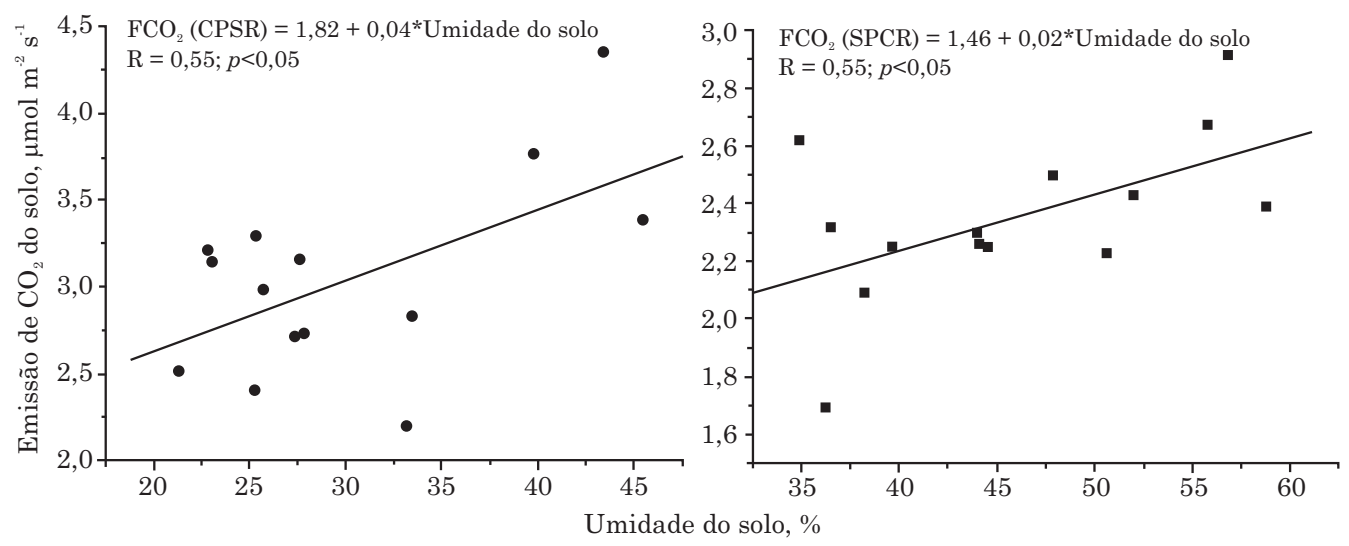

Figura 2. Correlação linear entre emissão de $\mathrm{CO}_{2}$ e umidade do solo nos manejos: sem preparo e com palha (SPCR) e com preparo e sem palha (CPSR).

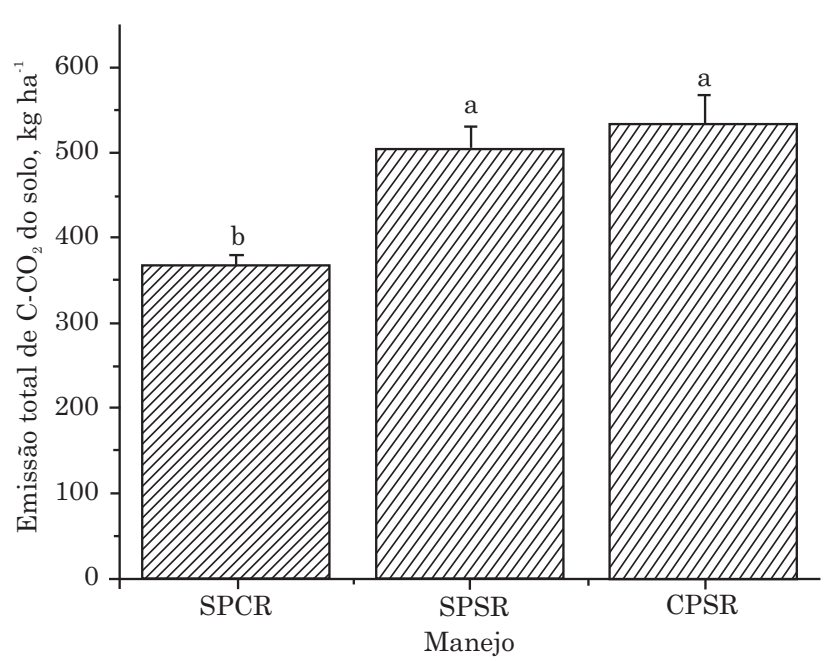

Figura 3. Emissão total de $\mathrm{C}-\mathrm{CO}_{2}$, com barras de erropadrão, nos diferentes manejos avaliados em 15 dias de estudo.

Comparando somente o efeito do preparo do solo entre os manejos SPSR e CPSR, observam-se valores muito semelhantes, indicando que, para as condições deste estudo, o preparo do solo não induziu a grandes perdas de C. Ao contrário deste estudo, vários autores reportaram a forte influência dessa prática de manejo nas perdas de $\mathrm{C}$ por erosão e em forma de $\mathrm{CO}_{2}$ emitido para a atmosfera (La Scala et al., 2006; Roscoe et al., 2006; Reicosky \& Archer, 2007; Gesch et al., 2007; Martínez-Mena et al., 2012).

Em adição, comparando-se os manejos SPCR e SPSR, a perda de C-CO $\mathrm{CO}_{2}$ do solo foi de $134 \mathrm{~kg} \mathrm{ha}^{-1} \mathrm{de}$ C- $\mathrm{CO}_{2}$ em 15 dias de medidas, o que representa $493 \mathrm{~kg} \mathrm{ha}^{-1}$ de $\mathrm{CO}_{2}$ emitido para a atmosfera, em razão da simples retirada da palha da superfície do solo. Figueiredo (2012), em estudo semelhante, observou que a retirada da palha durante a renovação do canavial resultou em emissões adicionais de $252 \mathrm{~kg} \mathrm{ha}^{-1} \mathrm{de}$ $\mathrm{CO}_{2}$, ou seja, emissões tão grandes quanto à queima prévia da palha anterior à colheita $\left(911 \mathrm{~kg} \mathrm{ha}^{-1} \mathrm{de}\right.$
$\mathrm{CO}_{2}$ ). Neste sentido, o autor discute que o efeito benéfico sobre as menores emissões de $\mathrm{CO}_{2}$ do solo com a eliminação da queimada em áreas de cana-deaçúcar pode ser anulado ao se retirar a palha remanescente da superfície do solo. Galdos et al. (2009), comparando os sistemas de colheita de cana queimada com cana crua, no sudeste do Brasil, também observaram menores emissões no sistema de cana crua, considerando o sequestro de $\mathrm{C}$ no solo.

\section{CONCLUSÕES}

1. Os manejos conservacionistas do solo em áreas de cana-de-açúcar com a manutenção dos resíduos sobre sua superfície, sem distúrbio, são estratégias eficientes para a redução das emissões de $\mathrm{CO}_{2}$ do solo. A cobertura vegetal propiciada pela palhada da cultura auxilia na preservação da umidade e favorece as menores temperaturas na superfície do solo.

2. O preparo do solo como fator isolado não influenciou em maiores taxas de emissão de $\mathrm{CO}_{2}$ desse. Os resultados mais expressivos referentes a elevações na emissão de $\mathrm{CO}_{2}$ e temperatura do solo foram observados quando a palha residual foi retirada dele.

\section{LITERATURA CITADA}

AMARAL, J.A.M.; MOTCHI, E.P.; OLIVEIRA, H.; CARVALHO FILHO, A.; NAIME, U.J. \& SANTOS, R.D. Levantamento semidetalhado dos solos do Campo Experimental de Dourados da Embrapa Agropecuária Oeste, município de Dourados-MS. Dourados, Embrapa Agropecuária Oeste/ Rio de Janeiro: Embrapa Solos, 2000. 68p.

CANELLAS, L.P.; BUSATO, J.G.; DOBBS, L.B.; BALDOTTO, M.A.; RUMJANEK, V.M. \& OLIVARES, F.L. Soil organic matter and nutrient pools under long-term non-burning management of sugar cane. Eur. J. Soil Sci., 61:375-383, 2010. 
CERRI, C.C.; MAIA, S.M.F.; GALDOS, M.V.; CERRI, C.E.P.; FEIGL, B.J. \& BERNOUX, M. Brazilian greenhouse gas emissions: The importance of agriculture and livestock. Sci. Agric., 66:831-843, 2009.

CERRI, C.E.P.; SPAROVEK, G.; BERNOUX, M.; EASTERLING, W.E.; MELILLO, J.M. \& CERRI, C.C. Tropical agriculture and global warming: Impacts and mitigation options. Sci. Agric., 64:83-99, 2007.

COMPANHIA NACIONAL DE ABASTECIMENTO - CONAB. Acompanhamento da safra brasileira: cana-de-açúcar, segundo levantamento, agosto/2012. Brasília. Conab. Disponível em: <http://www.conab.gov.br/OlalaCMS/ u $\begin{array}{llllllllllllllllll} & \mathrm{p} & \mathrm{l} & \mathrm{o} & \mathrm{a} & \mathrm{d} & \mathrm{s} & / & \mathrm{a} & \mathrm{r} & \mathrm{q} & \mathrm{u} & \mathrm{i} & \mathrm{v} & \mathrm{o} & \mathrm{s} & /\end{array}$ 12_09_05_09_11_59_boletim_cana_portugues__agosto_2012_2o_lev.pdf Safra 2010/2011>. Acesso em 31 out. 2012.

FIGUEIREDO, E.B. Balanço de gases de efeito estufa e emissões de $\mathrm{CO}_{2}$ do solo nos sistemas de colheita da canade-açúcar manual queimada e mecanizada crua. Jaboticabal, Universidade Estadual Paulista Júlio de Mesquita Filho, 2012. 111p. (Tese de Doutorado)

DENDOOVEN, L.; GUTIÉRREZ-OLIVA, V.F.; PATIÑOZÚÑIGA， L.; RAMÍREZ-VILLANUEVA， D.A.; VERHULST, N.; LUNA-GUIDO, M.; MARSCH, R.; MONTES-MOLINA, J.; GUTIÉRREZ-MICELI, F.A.; VÁSQUEZ-MURRIETA, S. \& GOVAERTS, B. Greenhouse gas emissions under conservation agriculture compared to traditional cultivation of maize in the central highlands of Mexico. Sci. Total Environ., 431:237-244, 2012.

EMPRESA BRASILEIRA DE PESQUISA AGROPECUÁRIA EMBRAPA. Sistema brasileiro de classificação de solos. Rio de Janeiro, Ministério da Agricultura e do Abastecimento, 2006. 306p.

GAISER, T.; STAHR, K.; BILLEN, N. \& MOHAMMAD, M.A.R. Modeling carbon sequestration under zero tillage at the regional scale. I. The effect of soil erosion. Ecol. Model., 218:110-120, 2008.

GALDOS, M.V.; CERRI, C.C. \& CERRI, C.E.P. Soil carbon stocks under burned and unburned sugarcane in Brazil. Geoderma, 153:347-352, 2009.

GESCH, R.W.; REICOSKY, D.C.; GILBERT, R.A. \& MORRIS, D.R. Influence of tillage and plant residue management on respiration of a Florida Everglades Histosol. Soil Till. Res., 92:156-166, 2007.

GRAHAM, M.H. \& HAYNES, R.J. Organic matter status and the size, activity and metabolic diversity of the soil microbial community in the row and inter-row of sugarcane under burning and trash retention. Soil Biol. Biochem., 38:21-31, 2006.

INTERGOVERNMENTAL PANEL ON CLIMATE CHANGE IPCC. Climate change 2007: The physical science basis. Summary for policymakers. Geneva, Switzerland, 2007. Disponível em: <http://www.ipcc.ch/ publications_and_data/publications_ipcc_fourth_ assessment_report_wg1_report_the_physical_science_basis.htm $>$. Acesso em: 26 set. 2012.
KANG, S.; DOH, S.; LEE, D.; LEE, D.; JIN, V.L. \& KIMBALL, J. Topographic and climatic controls on soil respiration in six temperate mixed-hardwood forest slopes, Korea. Global Change Biol., 9:1.427-1.437, 2003.

LA SCALA, N.; FIGUEIREDO, E.B. \& PANOSSO, A.R. A review on soil carbon accumulation due to the management change of major Brazilian agricultural activities. Braz. J. Biol., 72:775-785, 2012.

LA SCALA, N.; LOPES, A.; SPOKAS, K.; ARCHER, D.W. \& REICOSKY, D.C. Short-term temporal changes of bare soil $\mathrm{CO}_{2}$ fluxes after tillage described by ûrst-order decay models. Eur. J. Soil Sci., 60:258-264, 2009.

LA SCALA, N.; BOLONHEZI, D. \& PEREIRA, G.T. Shortterm soil $\mathrm{CO}_{2}$ emission after conventional and reduced tillage of a no-till sugar cane area in southern Brazil. Soil Till. Res., 91:244-248, 2006.

LOU, Y.; LIANG, W.; XU, M.; ELE, X.; WANG, Y. \& ZHAO, K. Straw coverage alleviates seasonal variability of the topsoil microbial biomass and activity. Catena, 86:117-120, 2011.

MARTÍNEZ-MENA, M.; LÓPEZ, J.; ALMAGRO, M.; ALBALADEJO, J.; CASTILLO, V.; ORTIZ, R. \& BOIXFAYOS, C. Organic carbon enrichment in sediments: Effects of rainfall characteristics under different land uses in a Mediterranean area. Catena, 94:36-42, 2012.

MARTINS FILHO, M.V.; LICCIOTI, T.T.; PEREIRA, G.T.; MARQUES JÚNIOR, J. \& SANCHEZ, R.B. Perdas de solo e nutrientes por erosão num Argissolo com resíduos vegetais de cana-de-açúcar. Eng. Agríc., 29:8-18, 2009.

MORELL, F.J.; ÁLVARO-FUENTES, J.; LAMPURLANÉS, J. \& CANTERO-MARTÍNEZ, C. Soil $\mathrm{CO}_{2}$ fluxes following tillage and rainfall events in a semiarid Mediterranean agroecosystem: effects of tillage systems and nitrogen fertilization. Agric. Ecosyst. Environ., 139:167-173, 2010.

PANOSSO, A.R.; MARQUES JR, J.; MILORI, D.M.B.P.; FERRAUDO, A.S.; BARBIERI, D.M.; PEREIRA, G.T. \& LA SCALA, N. Soil $\mathrm{CO}_{2}$ emission and its relation to soil properties in sugarcane areas under slash-and-burn and green harvest. Soil Till. Res., 111:190-196, 2011.

PANOSSO, A.R.; MARQUES JR, J.; PEREIRA, G.T. \& LA SCALA JR., N. Spatial and temporal variability of soil $\mathrm{CO}_{2}$ emission in a sugarcane area under green and slash-andburn managements. Soil Till. Res., 105:275-282, 2009a.

PANOSSO, A.R.; RIBEIRO, C.E.R.; ZANINI, J.R.; PAVANI, L.C.; PEREIRA, G.T. \& LA SCALA JÚNIOR, N. Variabilidade espacial da emissão de $\mathrm{CO}_{2}$, da temperatura e umidade de um Latossolo desprovido de vegetação sob diferentes lâminas de molhamento. Semina: Ci. Agron., 30:1017-1034, 2009b.

R DEVELOPMENT CORE TEAM. R: A language and environment for statistical computing. R Foundation for Statistical Computing. Vienna, 2011. ISBN 3-900051-070, URL <http://www.R-project.org/>.

RESENDE, A.S.; XAVIER, R.P.; OLIVEIRA, O.C.; URQUIAGA, S.; ALVES, B.J.R. \& BODDEY, R.M. Long-term effects of pre-harvest burning and nitrogen and vinasse applications on yield of sugarcane and soil carbon and nitrogen stocks on a plantation in Pernambuco, N.E. Brazil. Plant Soil, 281:339-351, 2006. 
REICOSKY, D.C. \& ARCHER, D.W. Moldboard plow tillage depth and short-term carbon dioxide release. Soil Till. Res., 94:109-121, 2007.

RONQUIM, C.C. Dinâmica espaçotemporal do carbono aprisionado na fitomassa dos agroecossistemas no nordeste do Estado de São Paulo. Campinas, Embrapa Monitoramento por Satélite, 2007. 52p.

ROSCOE, R.; BODDEY, R.M. \& SALTON, J.C. Sistemas de manejo e matéria orgânica do solo. In: ROSCOE, R.; MERCANTE, F.M. \& SALTON, J.C., eds. Dinâmica da matéria orgânica do solo em sistemas conservacionistas. Modelagem matemática e métodos auxiliares. Dourados, Embrapa Agropecuária Oeste, 2006. 304p.

SCHWARTZ, R.C.; BAUMHARDT, R.L. \& EVETT, S.R. Tillage effects on soil water redistribution and bare soil evaporation throughout a season. Soil Till. Res., 110:221$229,2010$.

SILVA-OLAYA, A.M.; CERRI, C.E.P.; LA SCALA JR., N.; DIAS, C.T.S. \& CERRI, C.C. Carbon dioxide emissions under different soil tillage systems in mechanically harvested sugarcane. Environ. Res. Lett., 8:1-8, 2013.

THORBURN, P.J.; MEIER, E.A.; COLLINS, K. \& ROBERTSON, F.A. Changes in soil carbon sequestration, fractionation and soil fertility in response to sugarcane residue retention are site-speciûc. Soil Till. Res., 120:99$111,2012$.
TOMINAGA, T.T.; CÁSSARO, F.A.M.; BACCHI, O.O.S.; REICHARDT, K.; OLIVEIRA, J.C.M. \& TIMM, L.C. Variability of soil water content and bulk density in a sugarcane ûeld. Aust. J. Soil Res., 40:605-614, 2002.

USSIRI, A.N. \& LAL, R. Long-term tillage effects on soil carbon storage and carbon dioxide emissions in continuous corn cropping system from an Alfisol in Ohio. Soil Till. Res., 104:39-47. 2009.

VARELLA, R.F.; BUSTAMANTE, M.M.C.; PINTO, A.S.; KISSELLE, K.W.; SANTOS, R.V.; BURKE, R.A.; ZEPP, R.G. \& VIANA, L.T. Soil fluxes of $\mathrm{CO}_{2}, \mathrm{CO}, \mathrm{NO}$ and $\mathrm{N}_{2} \mathrm{O}$ an old pasture and from native savanna in Brazil. Ecol. Applic., 14:221-231, 2004.

LAL, R. Challenges and opportunities in soil organic matter research. Eur. J. Soil Sci., 60:158-169, 2009.

PANOSSO, A.R.; PEREIRA, G.T.; MARQUES JÚNIOR, J. \& LA SCALA JR., N. Variabilidade espacial da emissão de $\mathrm{CO}_{2}$ em Latossolos sob cultivo de cana-de-açúcar em diferentes sistemas de manejo. Eng. Agríc., 28:227-236, 2008.

RAZAFIMBELO, T.; BARTHÈS, B.; LARRÉ-LARROUY, M.C.; DE LUCA, E.F.; LAURENT, J.Y.; CERRI, C.C. \& FELLER, C. Effect of sugarcane residue management (mulching versus burning) on organic matter in a clayey Oxisol from southern Brazil. Agric. Ecosyst. Environ., 115:285-289, 2006. 